\title{
Metabolic brain adaptations to recurrent hypoglycaemia may explain the link between type 1 diabetes mellitus and epilepsy and point towards future study and treatment options
}

\author{
Domenico Tricò $^{1}$ (D) $\cdot$ Raimund I. Herzog ${ }^{1}$
}

Received: 6 February 2017 / Accepted: 9 February 2017 / Published online: 24 February 2017

(C) Springer-Verlag Berlin Heidelberg 2017

Keywords $\beta$-hydroxybutyrate $\cdot$ Brain metabolism .

Epilepsy $\cdot$ Hypoglycaemia $\cdot$ Lactate $\cdot$ Type 1 diabetes

To the Editor: We read the retrospective study by Dafoulas, Toulis et al [1] on the association between type 1 diabetes and risk of epilepsy with great interest. In their article, the authors reported that individuals with type 1 diabetes are at approximately three-times greater risk of developing epilepsy compared with age- and sex-matched controls without type 1 diabetes. This finding, based on the data obtained from a large cohort of 24,610 individuals followed for a mean of 5.4 years, is robust and consistent with a recent study by Chou et al [2], also published in Diabetologia. Although the results of both studies may have substantial clinical implications, a better understanding of the causative mechanisms linking the two conditions will be essential to ultimately translate these findings into daily clinical practice. Potential mechanisms suggested by the authors include 'genetic or shared autoimmune factors' or 'cerebrovascular damage resulting from metabolic abnormalities' [1]. These factors are clearly good candidates for explaining the association between type 1 diabetes and epilepsy. Nonetheless, several additional key mechanisms that are linked to the incidence of recurrent hypoglycaemia (the most frequent complication of intensive insulin therapy in type 1 diabetes) might also contribute to this association.

Neuronal excitation is tightly tied to brain energy metabolism. Since the central nervous system mainly relies on

Raimund I. Herzog

raimund.herzog@yale.edu

1 Department of Internal Medicine, Yale University School of Medicine, 300 Cedar Street, P.O. Box 208020,

New Haven, CT 06520, USA glucose as its primary source of energy, severe hypoglycaemia represents a serious threat for normal brain metabolism and can alter the balance between inhibitory and excitatory neuronal networks, thereby increasing seizure susceptibility and the risk for brain damage. The acute functional and structural injuries induced by severe hypoglycaemia may persist even when normal glucose levels are restored, leading to permanent cognitive dysfunction, EEG abnormalities and, possibly, to a predisposition for unprovoked seizures [3].

To preserve brain metabolism from subsequent lowglucose events and prevent additional injuries, individuals with diabetes who experience frequent severe hypoglycaemia can develop cerebral adaptations in the uptake and utilisation of non-glucose energy substrates, such as lactate, which also affect neuronal metabolism at euglycaemia [4]. In fact, our group has demonstrated that the capacity of brain lactate uptake, as well as its cerebral oxidation, are enhanced in a model of recurrent hypoglycaemia [5]. In addition, we showed that elevated lactate is critical for maintaining neuronal metabolism and function under low-glucose conditions [5]. Based on the current understanding, we speculate that these chronic adaptations to hypoglycaemia could also support neuronal activity during seizures, thereby potentially explaining the association between type 1 diabetes and epilepsy. Indeed, during rapid neuronal firing, and particularly during seizures, the cerebral cortex requires a readily available energy supply to maintain its elevated metabolic requirements. In these circumstances, lactate has been increasingly recognised as the principal energy substrate [6]. In fact, Sada et al [7] recently reported that seizures and epileptiform activity can be reduced by inhibition of lactate dehydrogenase (LDH), a key enzyme of the astrocyte-neuron lactate shuttle, and that this effect is reversed by provision of pyruvate, its downstream metabolite. Altogether, these observations lead to the conclusion that adaptive mechanisms enhancing brain lactate transport and 
utilisation occur following recurrent insulin-induced hypoglycaemia, and may help initiate and sustain spontaneous seizures in individuals with type 1 diabetes.

The relevance of these two mechanisms (i.e. the potential for direct brain injury and harmful metabolic adaptations to be induced by recurrent hypoglycaemia) is further supported by the study from Chou et al [2] that identified an increased risk of epilepsy in patients with type 1 diabetes with a history of hypoglycaemia (HR 16.5 [95\% CI 5.19, 52.3] relative to participants without type 1 diabetes) compared with patients without hypoglycaemia (HR 2.67 [95\% CI 1.97, 3.62]). Unfortunately, the same association between epilepsy and incidence of hypoglycaemia was not investigated by Dafoulas et al [1] in their larger cohort of type 1 diabetes patients.

Further studies will be necessary to deepen our current understanding of the effect of type 1 diabetes mellitus on brain metabolism and to confirm the association between metabolic brain adaptations induced by recurrent hypoglycaemia and epilepsy. However, it is noteworthy that potential therapeutic implications of these observations exist. Our group has demonstrated that ketone bodies, which interestingly use the same transport system as lactate to cross the blood-brain barrier (monocarboxylate transporters), are effective in supporting synaptic transmission and preserving cognitive function under low-glucose conditions in type 1 diabetes [8]. Also, the ketogenic diet (i.e. a high-fat and low-carbohydrate diet favouring the endogenous production of ketones) has been successfully used for almost a century to treat children with drug-resistant epilepsy [6]. Thus, the dietary provision of small amounts of ketone bodies in individuals with type 1 diabetes may represent a promising therapeutic approach to support brain metabolism under hypoglycaemic conditions, while at the same time preventing associated brain injuries and reducing the risk of seizures in this vulnerable population.
Duality of interest The authors declare that there is no duality of interest associated with this manuscript.

Contribution statement Both authors conceived and wrote the article, revised it and approved the final version for publication.

Funding Work in the authors' laboratory is supported by the JDRF under 2-SRA-2014-271-M-R, as well as by the NIH/NIDDK via R01 DK101984, DP3 DK112227 and P30 DK045735.

\section{References}

1. Dafoulas GE, Toulis KA, McCorry D et al (2017) Type 1 diabetes mellitus and risk of incident epilepsy: a population-based, opencohort study. Diabetologia 60:258-261

2. Chou IC, Wang CH, Lin WD, Tsai FJ, Lin CC, Kao CH (2016) Risk of epilepsy in type 1 diabetes mellitus: a population-based cohort study. Diabetologia 59:1196-1203

3. Hyllienmark L, Maltez J, Dandenell A, Ludvigsson J, Brismar T (2005) EEG abnormalities with and without relation to severe hypoglycaemia in adolescents with type 1 diabetes. Diabetologia 48:412-419

4. Herzog RI, Sherwin RS, Rothman DL (2011) Insulin-induced hypoglycemia and its effect on the brain: unraveling metabolism by in vivo nuclear magnetic resonance. Diabetes 60:1856-1858

5. Herzog RI, Jiang L, Herman P et al (2013) Lactate preserves neuronal metabolism and function following antecedent recurrent hypoglycemia. J Clin Invest 123:1988-1998

6. Yang H, Wu J, Guo R et al (2013) Glycolysis in energy metabolism during seizures. Neural Regen Res 8:1316-1326

7. Sada N, Lee S, Katsu T, Otsuki T, Inoue T (2015) Epilepsy treatment. Targeting LDH enzymes with a stiripentol analog to treat epilepsy. Science 347:1362-1367

8. Page KA, Williamson A, Yu N et al (2009) Medium-chain fatty acids improve cognitive function in intensively treated type 1 diabetic patients and support in vitro synaptic transmission during acute hypoglycemia. Diabetes 58:1237-1244 